\title{
A Study of Point Laser for Automatic Local Road Condition Assessment: Feasibility, Challenges, and Limitations
}

\author{
Wenda Li \\ Is with the Department of Civil Engineering, University of Birmingham, \\ U.K. Email: w.li@bham.ac.uk
}

Michael Burrow

Is with the Department of Civil Engineering, University of Birmingham, U.K. Email:m.p.burrow@bham.ac.uk

Nicole Metje Is with the Department of Civil Engineering, University of Birmingham, U.K. Email:n.metje@bham.ac.uk

\section{Gurmel Ghataora*}

Is with the Department of Civil Engineering, University of Birmingham, U.K. Email: g.s.ghataora@bham.ac.uk

Abstract-Road condition assessment plays an important role in maintaining the integrity of mature and fast-expanding road networks. Such assessments provide invaluable information for local authorities that enable them to deliver timely preventative road network maintenance. To enable the best use of scarce maintenance resources, it is necessary to assess the condition of the roads periodically. In this article, we present the Point Laser (PL) system as an alternative approach for automatic road assessment. Compared to current systems, ground penetrating radar and the mobile laser scanning system, the PL system has the advantages of low hardware cost, low computational requirements, and fewer environmental restrictions 
in relation to its use. The article first outlines the principle of the PL system including a low-complexity signal processing approach. Afterward, the feasibility, challenges, and limitations of the PL system are discussed based on its use in a real environment. A visual-based road survey is used as a ground truth to determine the PL's performance in relation to the requirements of road condition assessment at various levels of road management. The results of the comparison demonstrate that the PL system is an ideal candidate for the automatic assessment of the condition of local road networks, especially those that require frequent assessment for road asset management.

| n most countries, local roads (i.e., urban and rural roads) constitute by far the largest proportion of the road network (typically more than $80 \%$ ), provide significant socioeconomic benefit, and constitute a significant asset value to a country. However, due to the combined damaging effects of traffic and the environment, local roads continually deteriorate and require periodic maintenance. Not only does maintenance sustain the value of local roads; it also helps to minimize road use costs that are directly related to road condition and thereby helps to ensure socioeconomic benefit at minimum user cost [1]. However, local road maintenance is often overlooked in favor of a country's high volume, strategic road network. To redress this balance there is a need to periodically assess local road condition to provide evidence for the need and benefit of their maintenance. Due to their vast extent, this should ideally be carried out by automated means. However, the automated assessment of local road condition is challenging because their predominant modes of deterioration, cracking and fretting (i.e., surface deterioration, which leads to potholes), are problematic to assess automatically in all weather conditions at normal traffic speeds. This difficulty is further exacerbated because of the variety of surface types that can be used to construct local roads. Consequently, traditionally the assessment of local road cracking and fretting is normally carried out visually by trained inspectors walking along the road or by using windshield surveys. However, the increasingly faster computing units, more advanced digital storage devices, and intelligent signal processing have the potential for both the quantitative and qualitative analysis of local road conditions at traffic speeds in real time and at relatively low cost [2].

A variety of vehicle-mounted sensors for automated road condition assessment have been investigated, including ground penetrating radar (GPR) [3], mobile laser scanning (MLS) [4], accelerometer [5], and vision system [6]. These have potential advantages over manual technologies, including improved repeatability, reproducibility, greater efficiency, and fast development cycles. For example, previous research on acoustic [7] and ultrasonic [8] systems (which transmit the mechanical wave from 50 $\mathrm{Hz}$ to $20 \mathrm{MHz}$ ) demonstrate the potential for nondestructive road condition assessment with acceptable resolution. In contrast, MLS is a more mature system and has been used for vehicle-based surveys [4], [9-11]. On the other hand, there are opportunities for the road condition assessment industry to learn from the rapid development of machine/ deep learning, vision systems that have become a critical component of the autonomous vehicles. Zhang et al. [12] developed a convolutional neural network for road cracking detection based on the images collected by a low-cost camera. A more advanced image system shown in Zhang et al. [13] can achieve pixel-level accuracy for extracting pavement defect to an accuracy of $90 \%$. Accelerometerbased approaches are affected by vehicle speed, mass, type, suspension system, and tire pressure [5]. The use of accelerometer-based approaches for the assessment of road surface cracking and fretting, the predominant modes of deterioration of local roads, is untried and would seem extremely challenging, and therefore research to date in their use has focused on assessing road roughness [5]. The limitations and restrictions of these systems are varied. Acoustic/ultrasonic systems are easily disrupted by air, gas, and water that restrict their potential application for high-speed surveying. The signal processing for MLS is usually highly complex and requires significant computational power. Furthermore, the research reported earlier [4], [9-11] has not demonstrated the ability for realtime processing. In addition, the powerful laser beam may be hazardous to passing pedestrians/animals. The signal processing for MLS is usually with high complexity that requires significant computational power, whereas the works mentioned earlier [4], [9-11] had not demonstrated the ability of real-time processing. The powerful laser beam may cause hazards to the surrounding pedestrians/ animals. In comparison, visual systems require suitable levels of road surface illumination; therefore, their use is constrained during inclement weather, and they often need large amounts of training data, increasing the costs of road surveys. These drawbacks significantly impinge on their use for extensive local road surveys where the weather can change rapidly, illumination levels vary, the types of defects vary, and the road surface types vary during the survey.

Current and future developments of onboard technologies for private vehicles include the adoption of both video 
cameras and inertial (accelerometer)-based equipment. While such equipment could be usefully used together with appropriate processing algorithms to assess road defects, such as roughness, rutting, and large areas of cracking, for the reasons outlined earlier, video and inertial-based systems are unsuitable for local roads where the assessment of fretting and areas of small cracks is of primary importance. Furthermore, the use of data from private vehicles to assess road condition relies on data being recorded on the same section of road by passes of multiple vehicles [5]. However, rural roads are often very lightly trafficked, and therefore such an approach would not be feasible.

In this article, we present an alternative approach for local road condition assessment that deploys a PL system that addresses many of the drawbacks of other systems described earlier. Furthermore, the PL system has the advantage of a relatively low-density of scanning data compared to the MLS system, thereby reducing computational power requirements, thus facilitating real-time processing. Furthermore, it costs considerably less than the MLS system and allows for rapid data update. The PL system has an excellent resolution in the longitudinal direction $(0.8 \mathrm{~mm}$ in this work) and is less hazardous to the surrounding environment. There has been little published work on the PL system. For example, [14] measures the road texture on the local roads, [15] assesses the road condition at the network level, and [16] uses the root-mean-square (RMS) value to estimate the change in road surface texture. In this work, a robust and low-complexity signal PL-based processing methodology has been presented that deals with noise and estimates road conditions to a resolution suitable for network-level analysis. The output is compared with manually inspected data to quantify the similarity. Afterward, the performance of the PL system is compared to another onboard MLS system to demonstrate the advantages and disadvantages of the proposed PL system for road condition surveys.

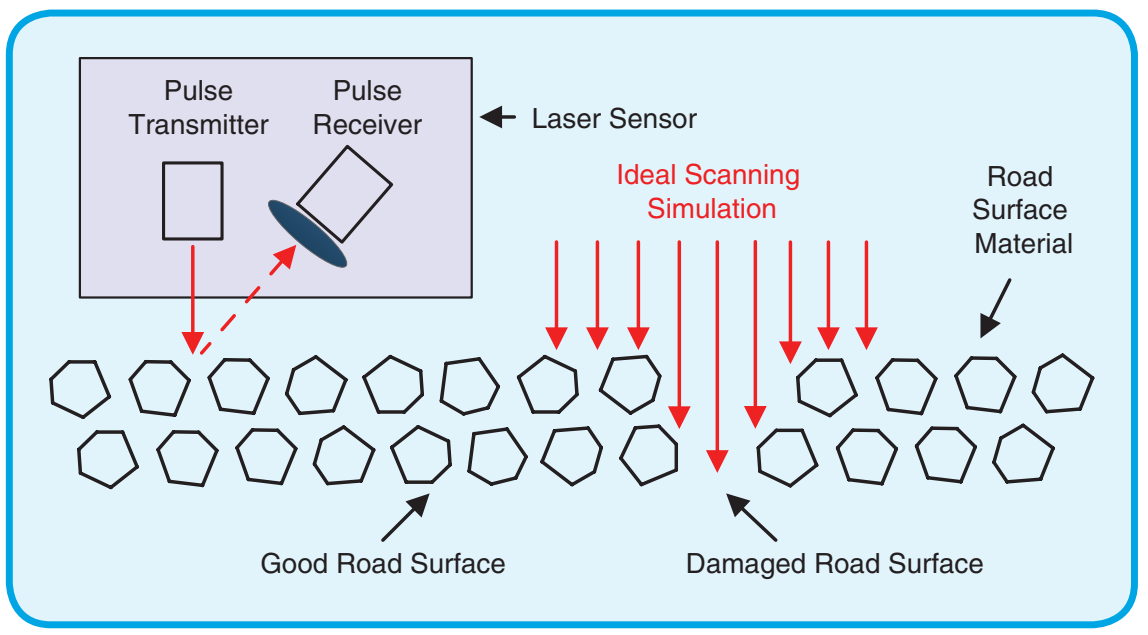

FIG 1 An overview of point laser for road condition assessment.

\section{Overview of Point Laser System}

\section{Principle of Point Laser}

A laser sensor continuously emits a pulse at a near-infrared wavelength, and part of the scattered reflected signal is captured by the sensor and digitized. Based on the travel time of the light, the relative distances between the laser and scanned objects can be calculated from the time delays between the pulse transmission and return. In practice, the laser sensor utilizes optical 2D triangulation as shown in Figure 1, whereas the angle of the reflected pulse depends on the distance. Unlike most commercial MLS systems, the PL system does not scan the entire road surface but only the longitudinal direction (i.e., in the direction of travel). As a result, each PL sensor outputs a 2D profile of the road surface instead of the 3D point cloud data, which means the down-conversion process is not required. This enables much lower computational complexity when compared to the 3D MLS point clouds technique or the 2D georeferenced feature (GRF) images [17].

A road is typically constructed of several layers of materials. Over time, due to the damaging effects of traffic and the environment, the road surface loses aggregate particles as the binder ages, causing a consequential loss of adhesion (this is known as fretting). A PL sensor can detect this loss of the aggregate due to its high resolution. An idealized scanning simulation for a PL sensor is shown in Figure 1. For a sound road surface, the amplitude and frequency of the reflected laser signal are relatively constant, whereas for a damaged road surface, the reflected signal is of much greater variation in terms of amplitude and frequency. Thus, it is possible to assess the quality of the road surface by using information regarding the reflected amplitude and frequency of the signal. However, in reality, noise from multiple sources, for example the acceleration from vehicle and the change in the surface texture of the road, induce significant interference. For accurate road condition assess, such noise needs to be removed before processing. Details of how this has been achieved in the proposed system are described in the "Preprocessing" section.

\section{System Design}

The PL system is mounted on an inservice road condition survey vehicle (a Ford TDCi model), namely, Highways England's Road Research Information System 2 (HARRIS2) [15]. The system consists of three main components: sensors for road condition assessment, a system for providing location information, and a central control unit. 


\section{Scanning Sensors}

HARRIS2 contains five ProfiCura 2D point lasers [18] designed for operating in the harsh road environment. Each laser is aligned at 90 degrees to the road surface, and the lasers are distributed horizontally across the vehicle. A close view of the PL system is shown in Figure 2. HARRIS2 is also equipped with a Laser Crack Measurement System (LCMS) [19] with high resolution in the transverse direction that is capable of taking 1,000 transverse profile measurements per second across a width of approximately $4.2 \mathrm{~m}$. The LCMS collects the 3D laser data from the road surface and converts it into 2D georeferenced images, and it is operated simultaneously with the PL system for comparison purposes.

Table 1 compares 2D profile data along with 2D image-driven and 3D MLS point-drive extraction road condition monitoring systems. The PL sensor used in this work outputs a $2 \mathrm{D}$ profile data with the lowest data dimension compared to the other laser techniques [3], [19-21]. Also, a low-complexity histogram analysis [22] has been used to process the profile data. In comparison, converting the 3D MLS point clouds into 2D images is an effective solution to overcome inconsistency and variance issues in intensity that can occur due to the shape of road objects. This approach also reduces the dimensions of the data and the need to use very robust deep learning algorithms to analyze the data. In comparison, the 3D point cloud-based systems [20] can improve the accuracy of assessing complex types of road defects. However, it is a challenging task to extract from the large-volume MLS point clouds especially for unevenly distributed point clouds and complex concavoconvex features [17].

\section{Navigation and Orientation System}

An important component of automated systems used to assess road condition is location referencing. HARRIS2 uses a global navigation satellite system (GNSS), which uses a GPS signal to provide the location information in the form of an Ordnance Survey grid reference (OSGR) (OSGR is a grid references system used in the United Kingdom). The positioning accuracy of this system is affected by multipath propagation issues (for example, tunnels, buildings, and trees). To eliminate such effects and bypass the limitation of GPS signal, an inertial measurement unit (IMU) is used to provide the instant velocity, position, and attitude measurements. The IMU provides continuous positioning information in the case of GNSS malfunction. In addition, the IMU contains three accelerometers and three gyroscopes, which can also be used for acceleration and angular measurements. The resulting overall localization accuracy is approximately $1 \mathrm{~cm}$ horizontally and $2 \mathrm{~cm}$ vertically with respect to local GNSS-reference stations [17].

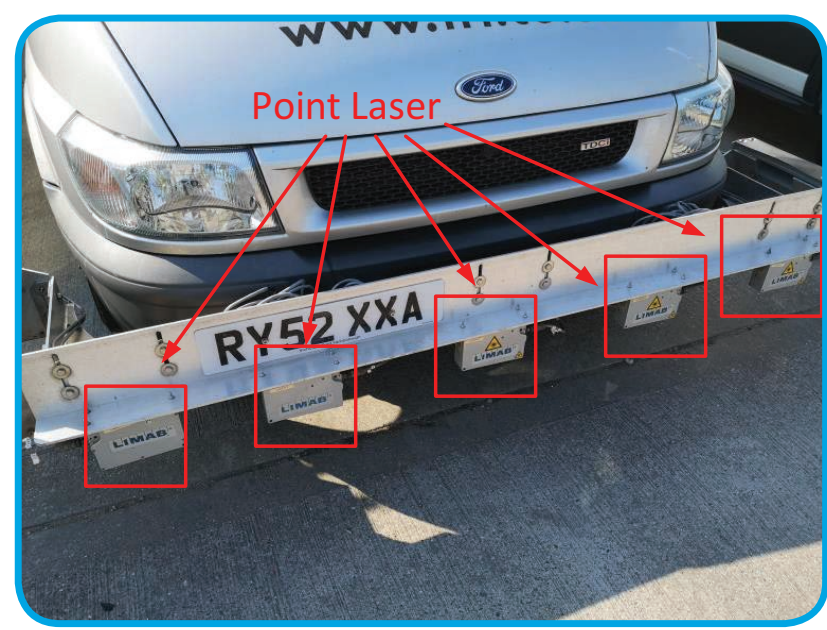

FIG 2 The point laser system mounted on the HARRIS2.

Table 1. Comparison table of laser systems.

\begin{tabular}{|c|c|c|c|}
\hline Systems & Point Laser & Onboard MLS & $\begin{array}{l}360 \text { Degree } \\
\text { MLS [20] }\end{array}$ \\
\hline $\begin{array}{l}\text { Longitudinal } \\
\text { Resolution }\end{array}$ & $0.803 \mathrm{~mm}$ & $4 \mathrm{~mm}$ & $8 \mathrm{~mm}$ \\
\hline $\begin{array}{l}\text { Transverse } \\
\text { Resolution }\end{array}$ & $\mathrm{NaN}$ & $1 \mathrm{~mm}$ & $8 \mathrm{~mm}$ \\
\hline Vertical Resolution & $0.01 \mathrm{~mm}$ & $1 \mathrm{~mm}$ & $8 \mathrm{~mm}$ \\
\hline Categories & $\begin{array}{l}\text { 2D Profile } \\
\text { data }\end{array}$ & $\begin{array}{l}\text { 2D } \\
\text { Georeferenced } \\
\text { image }\end{array}$ & $\begin{array}{l}\text { 3D Point cloud } \\
\text { data }\end{array}$ \\
\hline $\begin{array}{l}\text { Signal Processing } \\
\text { Method }\end{array}$ & $\begin{array}{l}\text { Histogram } \\
\text { analysis }\end{array}$ & $\begin{array}{l}\text { Hough } \\
\text { transformation }\end{array}$ & $\begin{array}{l}\text { Deep learning } \\
\text { neural network }\end{array}$ \\
\hline $\begin{array}{l}\text { Measurement Rate } \\
\text { (max) }\end{array}$ & $2 \mathrm{k} / \mathrm{sec}$ & $28 \mathrm{k} / \mathrm{sec}$ & $550 \mathrm{k} / \mathrm{sec}$ \\
\hline Information & $\begin{array}{l}\text { Network-level } \\
\text { information }\end{array}$ & $\begin{array}{l}\text { Road markings, } \\
\text { road crackings }\end{array}$ & $\begin{array}{l}\text { Multiple } \\
\text { transportation } \\
\text { information }\end{array}$ \\
\hline Real-Time Ability & $\begin{array}{l}\text { Onboard } \\
\text { processing }\end{array}$ & $\begin{array}{l}\text { Offline } \\
\text { processing }\end{array}$ & No demonstration \\
\hline
\end{tabular}

\section{Central Control Unit}

HARRIS2 also contains a central control unit (a computer server), a highly integrated control system designed to control all onboard sensors (e.g., laser system, MLS), synchronize the data from each system with a time stamp, and store the location information from the GNSS system. Further information about the HARRIS2 central control unit can be found in [15].

\section{Signal Processing of Point Laser}

This section presents the signal processing for the proposed PL system that is used to 1) remove noise and 2) analyze the processed signal to estimate road condition. The 
structure of the developed signal processing is shown in Figure 3.

\section{Preprocessing}

It was observed that several sources of noise can affect significantly the PL data, and such noise therefore needed to be filtered at the preprocessing stage. To illustrate this, an example of 50-meter raw data points is provided in Figure 4(a).

\section{Sensor Noise}

Sensor noise is a result of the PL sensor recording an erroneous data point due to the internal clock issues (signal source inside PL sensor) providing an incorrectly measured time of flight between the outward and reflected laser pulses. By analyzing the entire data set from a survey, the erroneous data point typically accounts for approximately $0.5 \%$ of the data. Since the longitudinal resolution of the PL sensor is $0.803 \mathrm{~mm}$, this means around six erroneous data points per meter. These erroneous data points are of much higher amplitude [as shown in Figure 4(a)] and can cause significant interference for the later histogram analysis.

In this work, a two-step algorithm was applied to remove the erroneous data points while not affecting the other authentic data points. First, an adaptive threshold $T(t)$ based on a moving average of minima (MAM) method [23] was used to identify the erroneous data points. $T(t)$ is calculated as follows:

$$
T(t)=\sum_{i=-\frac{1}{2} l_{n}}^{\frac{1}{2} l_{n}} c_{i} y(t+i)+w
$$

where $y(t)$ represents the collected data points, $l_{n}$ is the window length, $c_{i}=1 /\left(l_{n}+1\right)$ is the weighting factor, and $w$ is a constant. Data points above the threshold are considered to be erroneous [e.g., the red line in Figure 4(a)]. The second step replaces erroneous data points with the mean value of authentic adjacent data points. In the example given in Figure 4(a), the resulting data points are shown in Figure 4(b).

\section{Vehicle Dynamic}

Another major source of interference is from vehicle vibrations, which are particularly prevalent during acceleration and deceleration. Since the laser sensor measures the relative distance to the road surface, a change in vehicle height may affect the measurement of road defects. In this work, the vehicle dynamic issue was regarded as a baseline correction problem since the motion introduces components that have significantly greater fluctuations in both magnitude and wavelength when compared to those resulting from both the road texture and the defects. The MAM algorithm described in (1) was used again for the baseline estimation (albeit with a different window length). Accordingly, the resulting corrected data points $\hat{y}(t)$ are calculated as

$$
\hat{y}(t)=y(t)-y^{V}(t),
$$

where $y^{V}(t)$ is the estimated baseline. The result of baseline correction is shown in Figure 4(c), from which it can be seen that the major component due to the vehicle dynamic has been successfully corrected. For example, the troughs (e.g., near 10 and 40 meters) can be easily observed following the baseline correction.

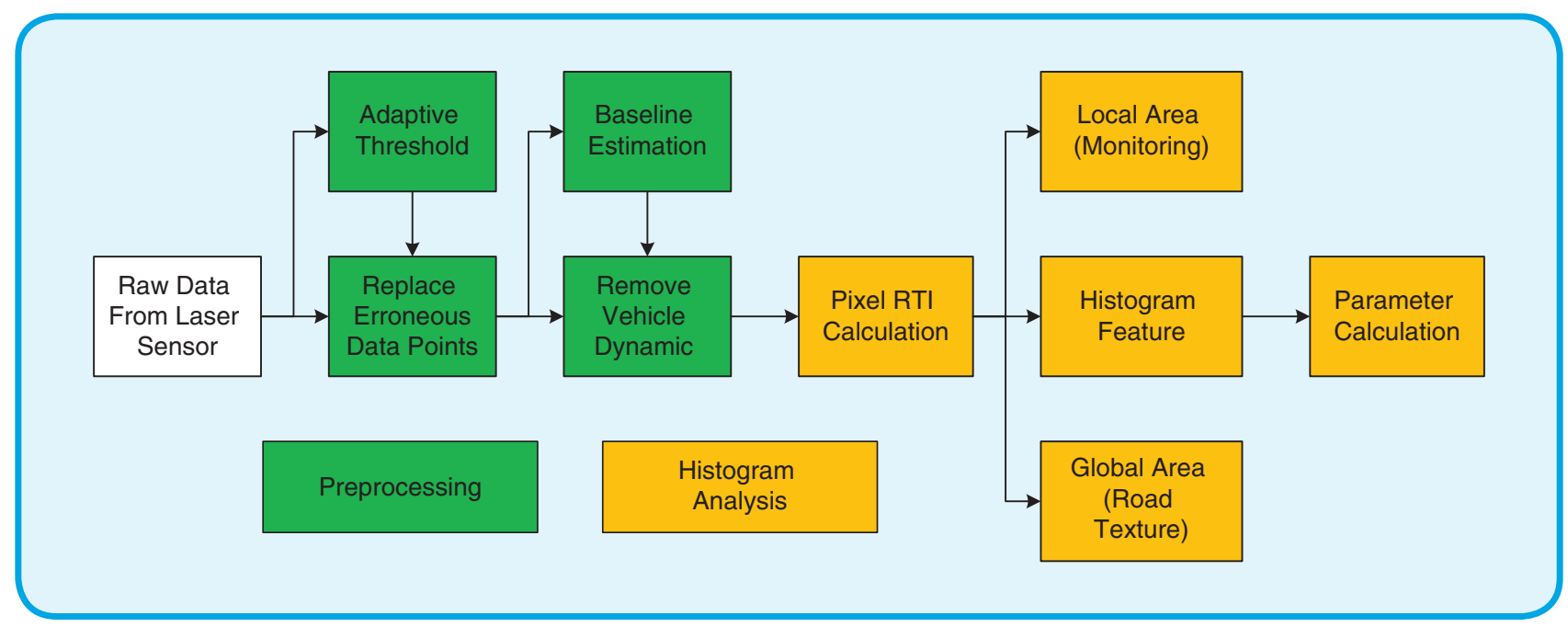

FIG 3 A block diagram of signal processing for point laser. 


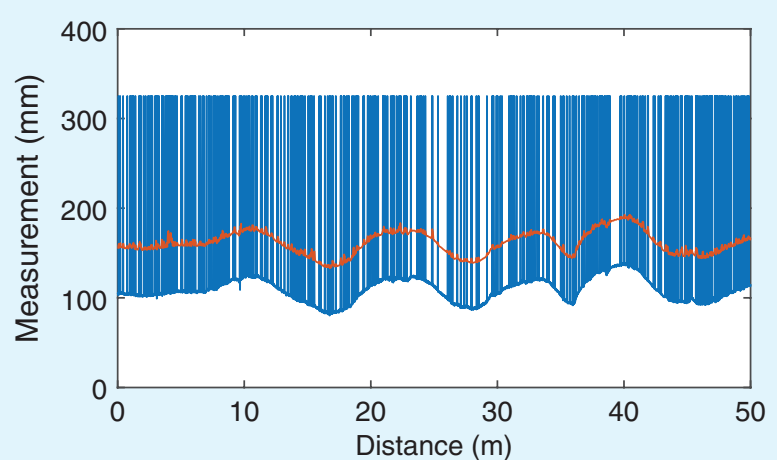

(a)

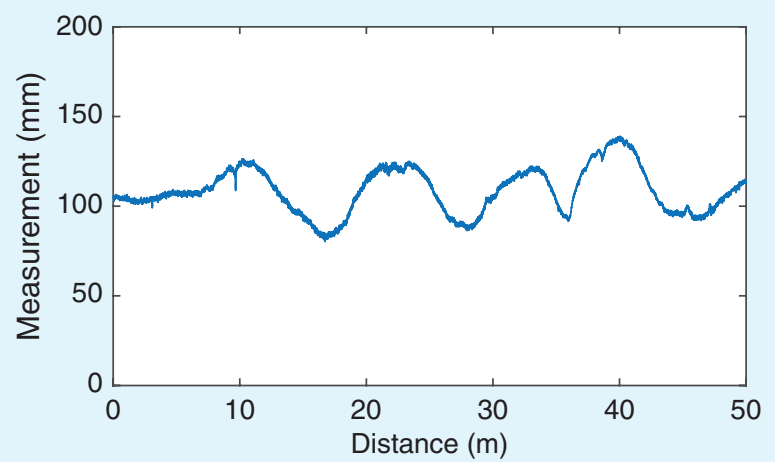

(b)

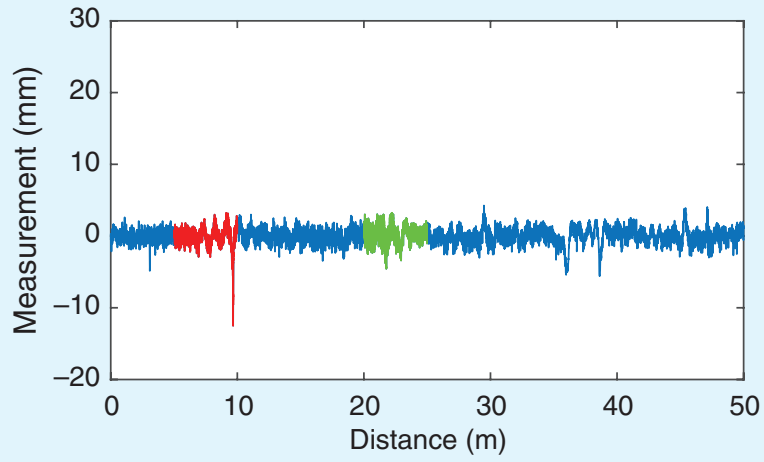

(c)

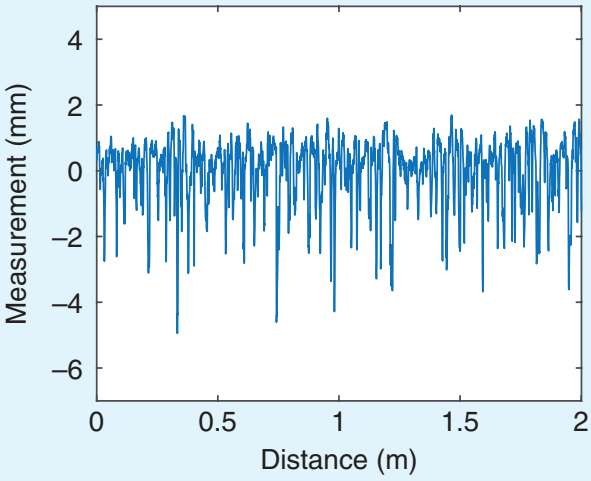

(d)

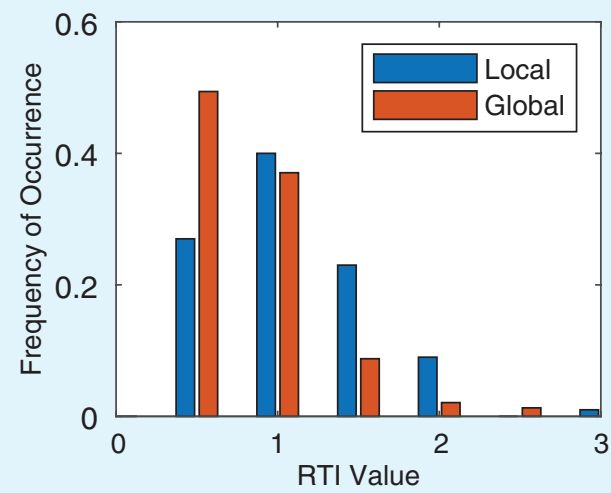

(f)

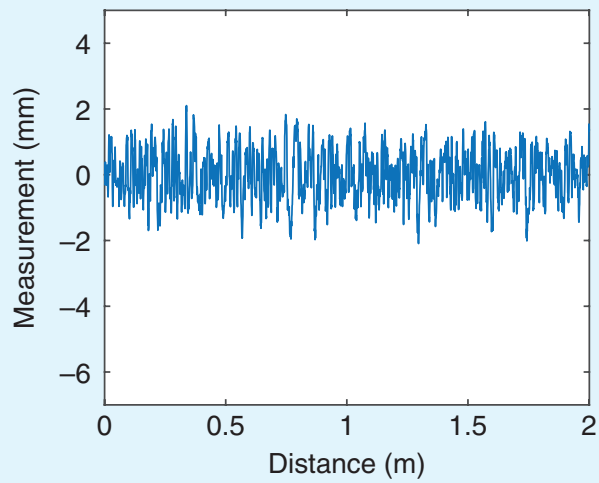

(e)

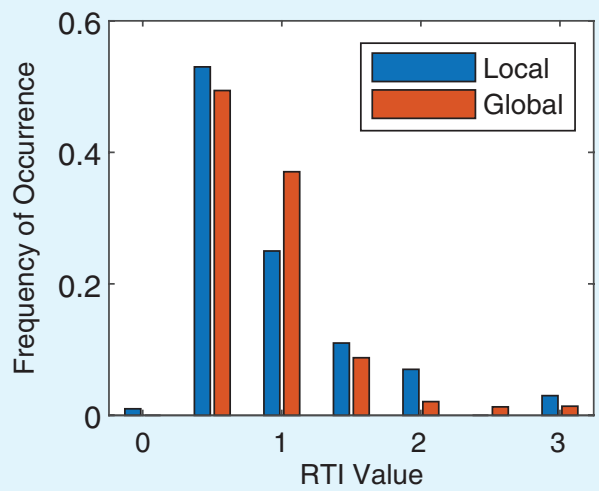

(g)

FIG 4 A demonstration of the signal processing for a PL system: (a) raw data points (the red line is adaptive threshold); (b) after sensor noise removal; (c) after baseline correction (the red part is road surface containing fretting; the green part is a sound road surface); (d) and (e) measurements of two types of road texture; (f) histogram analysis of fretting road surface; and $(\mathrm{g})$ histogram analysis of smooth road surface. 


\section{Road Condition Estimation}

Another issue that needs to be considered is the changes in road surface texture that are likely to be encountered by a survey vehicle during a survey. Different road textures can have different sizes of surface aggregate, and as a result, this can cause issues in the measurement of fretting at the point when the vehicle traverses two roads with different surface textures. Examples of two different road textures are shown in Figure 4(d) and (e).

\section{Road Texture Index Calculation}

The Root Texture Index (RTI) was adopted as a measure of road texture as discussed in our previous work [22], [24]. RTI evaluates the changes in road surface measurement for a given segmentation with respect to the RMS value. The $i$ th RTi value is calculated as

$$
R T I_{i}=\sqrt{\frac{1}{n_{s}} \sum_{k=1} n_{s} y(k)^{2}},
$$

where $n_{s}$ is the number of data points per segmentation.

\section{Global and Local Area}

In this work, the concept of the global area (i.e., an estimate the type of road texture over a relatively long distance as a reference) and the local area (i.e., the scanning area) is used [22]. The concept assumes that the road texture remains unchanged over the long distance (global area) when compared to the part of road being assessed (i.e., the local area). By doing so, the system can analyze the types of road texture without prior knowledge of the texture features. The relationship between the number of data points in global area $n_{g}$, the local area $n_{l}$, and the segmentation length $n_{s}$ follows $n_{g}>n_{l} \gg n_{s}$.

As a result, the number of RTI values in a global area is greater than that in a local area, and, consequently, frequency histograms of local and global RTI values are compared to assess the condition of the local area compared to the global area. Here, two examples of histogram analysis are provided based on the data points in Figure 4(c). The section from $5 \mathrm{~m}$ to $10 \mathrm{~m}$ shows an area of road surface that has significant fretting (the red part) whereas the section from $20 \mathrm{~m}$ to $25 \mathrm{~m}$ is a sound road surface (the green part). The output histograms are provided in Figure 4(f) and $(\mathrm{g})$, respectively. As can be seen, the histogram of the sound road surface has a similar distribution to that of the global area, whereas the fretted road surface shows more variance.

\section{Parameter Calculation}

The final stage is to generate meaningful parameters that can present the difference between histograms of a fretted and sound road surface meaningfully. Histogram features can be considered to be vectors; thus, the problem can be considered to be one of understanding the difference/ similarity of the vectors. Two parameters were chosen to this end:

- Mean-square-error (MSE). MSE represents the difference between the RTI distribution of the local and surrounding global lengths. A low MSE value occurs if the distributions of local and global RTI values are similar, and thus there is a low amount of fretting in the local length. MSE is calculated as $M S E=1 / n \sum_{i=0}^{n}\left|h_{g_{i}}-h_{l_{i}}\right|$, where $h_{g_{i}}$ and $h_{l_{i}}$ are the histogram features from global and local area.

- Correlation-coefficient $(R)$. $\mathrm{R}$ is a measure of the similarity between the frequency distribution of the RTI values of the local length and that of the global length. Low $\mathrm{R}$ values indicate that the local RTI distribution is significantly different compared to the global RTI distribution, and therefore the local length of road contains large amounts of fretting. $\mathrm{R}$ is calculated as $R=\sum_{i=0}^{n} h_{g_{i}} h_{l_{i}} / n \sigma_{h_{g}} \sigma_{h_{l}}$, where $\sigma$ represents the standard deviation.

The efficacy of these processes is discussed in the next section.

\section{Observations and Discussions}

In this section, we discuss the detection performance of the system outlined earlier, using data collected from a number of road surveys. Three challenges are outlined together with their possible solutions. Limitations are also discussed to present the boundaries of using the PL system for road condition surveys.

\section{Feasibility}

The feasibility of the PL system is demonstrated by comparing data collected from four roads near Crowthorne, United Kingdom, surveyed by HARRIS2 with the Detailed Visual Inspection (DVI) [25] of the roads carried out by trained surveyors. The DVI survey classifies road surface fretting in terms of high (H), moderate (M), and low (L) levels. A brief description of the data set is provided in Table 2 .

The MSE value against the $R$ value for each road is plotted in Figure 5 to visualize the algorithm's output. Each point represents the PL measurement over a $5 \mathrm{~m}$ distance. Sound road surfaces should have small MSE value (i.e., close to 0 ) and a high $\mathrm{R}$ value (i.e., close to 1 ), whereas damaged road surface will have a high MSE and a low $R$ value. In other words, in Figure 5, data points in top left corner represent road sections with low amounts of fretting area, and those in bottom right corner represent road sections with a high amount of fretting. Figure 5 presents the average condition of each road. For example, the majority of sections of road one are in generally good condition with most data points concentrated in the top left corner. On the other hand, the measured sections in road four are scattered across the bottom right corner, suggesting the road is in poor condition. In contrast, roads two and three have 
more points distributed among the middle area, indicating that the road sections are in moderate condition. By inspection, these results match the DVI data shown in Table 2. There are also some anomalies, for example, the point (MSE $=0.06$ and $R=0$ ) in the right bottom of Figure 5(c). However, the number of anomalies is very small, and they do not affect significantly the assessment of the average condition of the entire road. Even in roads three and four, there are a number of sections with low MSE and high $\mathrm{R}$ values.

The MSE and R values shown in Figure 5 are presented in Figures 6 and 7 in the form of histograms of the normalized frequency of occurrence. These histograms show clearly trends in condition for each road. The majority of MSE values for roads one and two lie in the range between 0.01 and 0.02 with $\mathrm{R}$ values between 0.99 and 0.97 . This suggests that both roads are on the whole in good condition and correspond to their actual road condition. In comparison, the majority of MSE values from road four are between 0.03 and 0.04 , and most $R$ values are less than 0.90 , indicating a damaged road section. This finding is verified by the actual condition of the road given in Table 2 .

\begin{tabular}{|c|c|c|c|c|}
\hline Road & Description & Length & Class & Fretting Level \\
\hline One & Byron Drive & 0.421 & Secondary road & $\mathrm{L}$ \\
\hline Two & Duke's Ride & 0.480 & B road & $\mathrm{L} / \mathrm{M}$ \\
\hline Three & $\begin{array}{l}\text { Wokingham } \\
\text { Road }\end{array}$ & 0.711 & A road & M \\
\hline Four & Hanworth Road & 1.337 & Secondary road & $\mathrm{H}$ \\
\hline
\end{tabular}

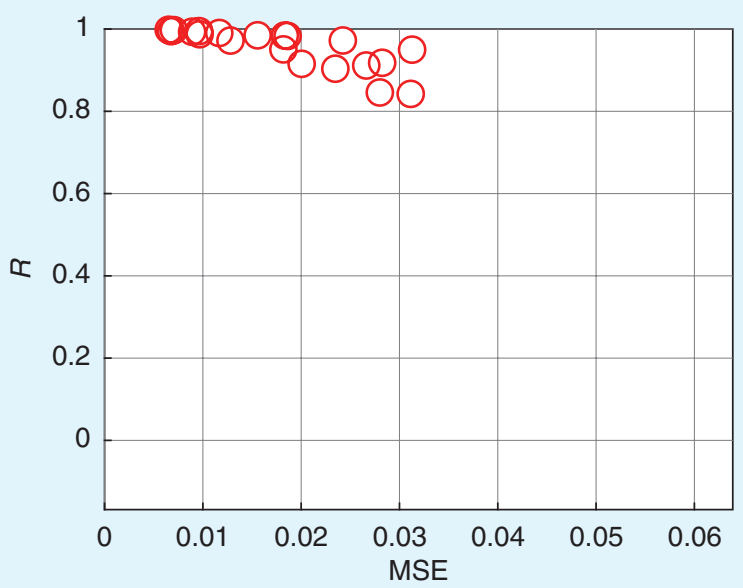

(a)

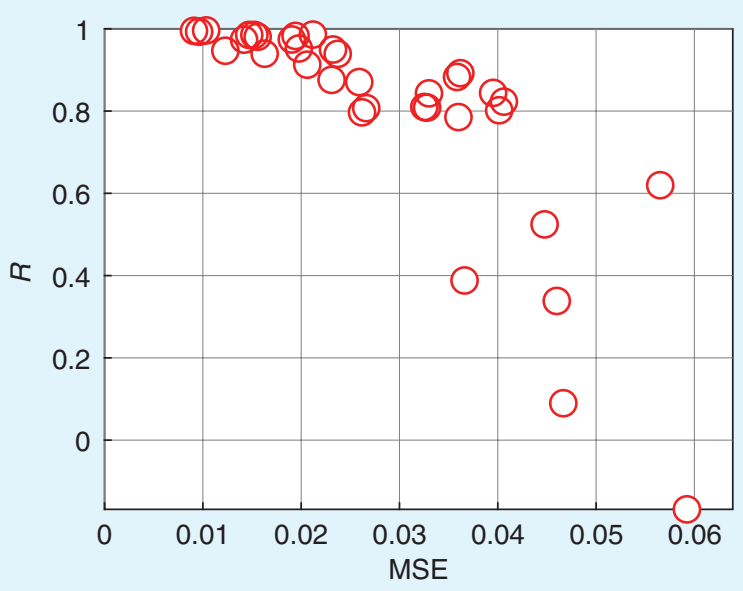

(c)

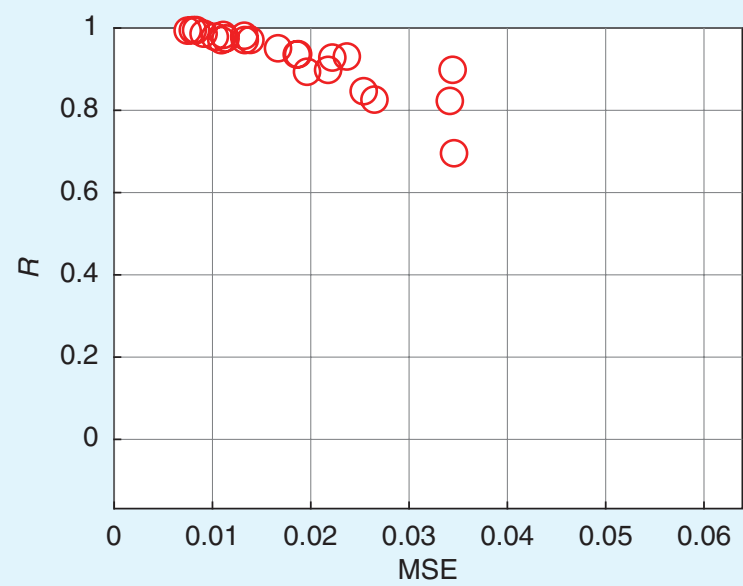

(b)

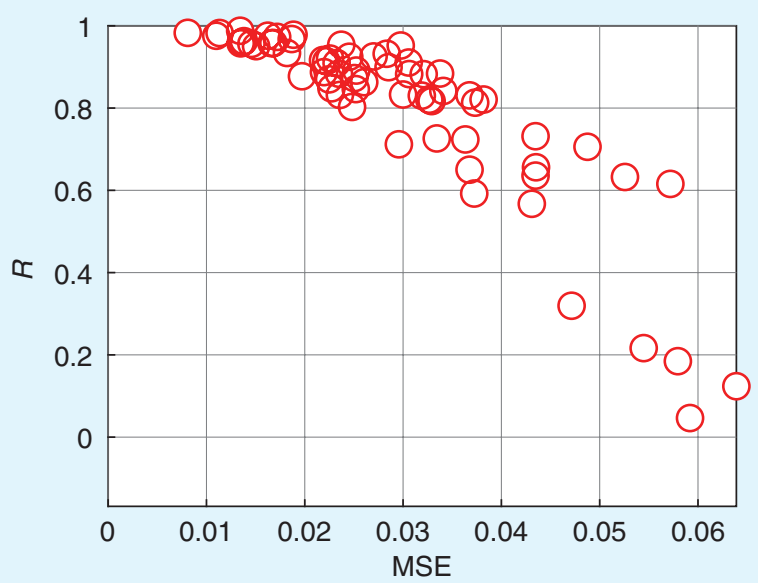

(d)

FIG 5 An MSE and R plot for each road survey: (a) road one, (b) road two, (c) road three, and (d) road four. 
Road surveys can be considered to support three levels of road asset management decision making, namely, strategic planning, tactical programming, and operations [26]. The data requirements to support each level of decision making are different and can be defined according to the World Bank's information quality level (IQL) approach [27]. A paired difference test [28] was used to further compare statistically the results of the PL system and the DVI methodology for each of the three levels of management. Figure 8 provides the results of strategic planning $(500 \mathrm{~m})$, tactical programming $(60 \mathrm{~m})$, and operations $(20 \mathrm{~m})$. The PL system and DVI show similar results for all three levels of road management. In particular, the similarity is highest when the data are considered over a 500-m length of road. Data to this level of detail are at IQL 3/4 and are suitable for strategic road asset management decision making [22]. This means the current approach is sufficient for strategic, or network-level, road condition analysis. Accordingly, this data would be useful to support inspection required to determine periodical maintenance and infrastructure construction planning.

\section{Challenges}

\section{Unexpected Measurements}

Apart from the interference described in the "Preprocessing" section, there are a number of issues that may po-

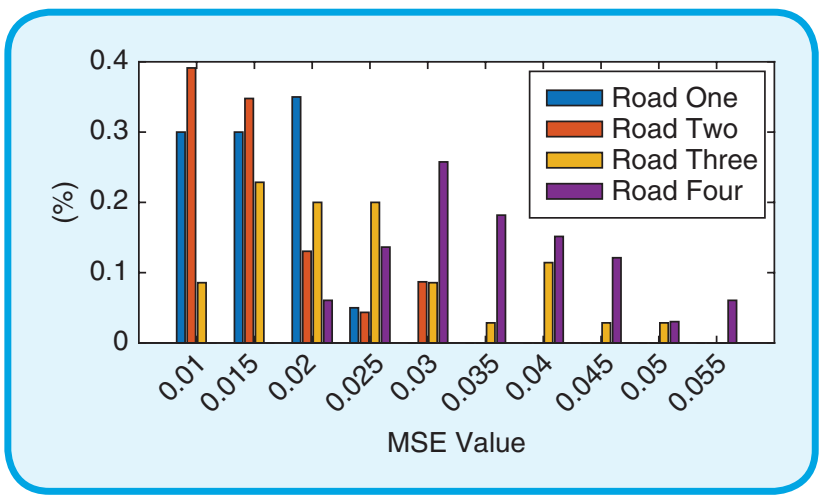

FIG 6 A histogram on MSE values from four road surveys.

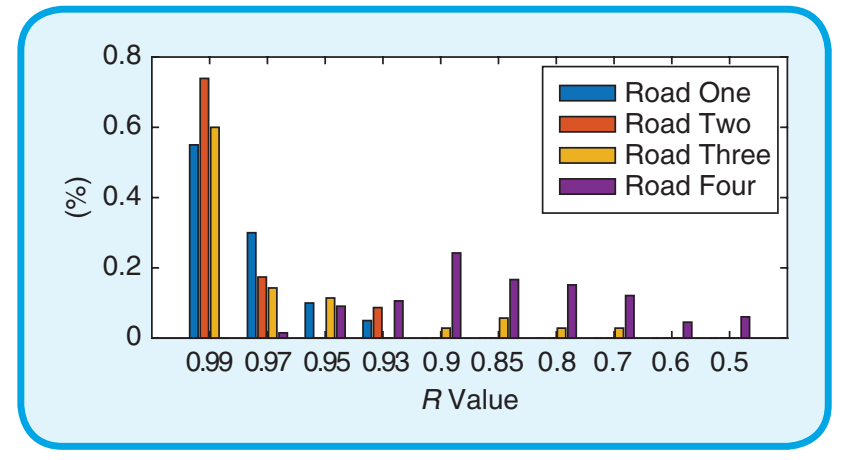

FIG 7 A histogram on $R$ values from four road surveys. tentially affect the system's performance. Four different types of interference are shown in Figure 9 (the sensor noise has been removed) varying from a few millimeters to more than hundred meters in extent and with a variety of magnitudes. For example, data points in Figure 9(a) show a saturated and a constant gap spanning over one meter, whereas in Figure 9(b) a deep trough of more $50 \mathrm{~mm}$ in depth and $0.1 \mathrm{~m}$ in width is apparent at a distance of 0.4 $\mathrm{m}$. In comparison, the apparent anomalies in Figure 9(c) and (d) are similar to the road measurements in terms of the magnitude and variation, but with a unique periodicity.

The cause for these anomalies varies. Sensor error can explain the measurements in Figure 9(a) and (b) as they are of constant amplitude for a period of time, which should not be a case when scanning a road section. For Figure 9(c) and (d), small objects on the road surface may be the reason for those measurements, for example, when the scan is over a manhole cover or a road marking. Undoubtedly, these abnormal measurements will affect the RTI calculation and the histogram analysis. In the "Feasibility" section, we have demonstrated that with the current setup, the system output can achieve the requirement of IQL 3/4 for network-level analysis. But to achieve a higher level in IQL $1 / 2$, these irregular measurements need to be removed.

Anomalies such as those in Figure 9(a) and (b) are easy to detect, as they have very different magnitudes when compared to the regular road surface. Algorithms such as those proposed by [29] could be suitable to identify such anomalies. The challenge is more complicated for the data points in Figure 9(c) and (d), as they may appear more frequently. Their detection and removal require training a classifier with a data set labeled as "normal" and "abnormal" so that the system can separate the normal and abnormal measurements.

\section{Road Surface Texture}

Another challenge is the effect of the change of road texture, which can occur during a road condition survey. An example of measurements on two road textures using an MLS and PL sensors is shown in Figure 10. As can be seen, the two types of road texture are clearly distinguishable in the 2D image from the MLS with different intensity. In comparison, the difference between two textures is not as apparent in the PL measurement, but it still can be observed that the data points from road texture A contain more fluctuations than for texture B. Despite the use of the local and global concept described earlier to negate the need to determine prior to the survey the type of road texture, a larger window than that used for the earlier analysis is required to show the changes in texture. This potentially reduces the accuracy of the output as road defects vary in size depending on the road texture, while the inaccurate texture estimation reduces the number of road defects detected. 
The low dimension of data points in a PL survey restricts the estimation of road texture. A sliding window could help to improve the sensitivity by generating more estimations over a given area. However, the global area remains a network-level method as it contains a large number of data points that are insensitive to a sudden change and enables changes in road texture to be considered automatically. On the other hand, the analysis of very small sections of the road surface can significantly increase the accuracy of texture measurement by examining the characteristics of each data point. Such an approach will require significant additional computational power due to the larger number of data points that need to be assessed. Thus, the tradeoff between the computational power and accuracy required to measure texture needs to be carefully considered and assessed according to the IQL required.

\section{Imperfect Vehicle Dynamic Cancellation}

As described in the "Preprocessing" section, an MAM filter was used for simple and low-complexity baseline correction. However, the varied dynamic motions of the vehicle mean a single MAM filter cannot accurately remove all of the dynamic components, and as a result, those components that are not removed can strongly affect the histogram analysis.
Improvements can be made to both the software and hardware to better account for the effects of vehicle dynamics. From the software side, filters with high-resolution spectra that are able to process multivariate can provide better performance in baseline correction [30]. But the application of such filters can significantly increase the processing time and the choice of appropriate filter parameters is critical. On the hardware side, the onboard IMU provides the inertial information about the vehicle movement. Real-time vehicle dynamics can be calculated by using this information, allowing their effect to be removed from the PL measurement with a high degree of confidence.

\section{Limitations}

Since the PL sensor scans only in the longitudinal direction, it has a number of limitations when compared to an MLS system. Based on the analysis of the data obtained from the field trial, it was observed that several road defects are hard to identify by the PL system because their shape or location are not within the scanning area. Also, low-level road surface associated features (when compared to 3D point cloud data) are apparent in the PL measured data that are problematic for defect classification.

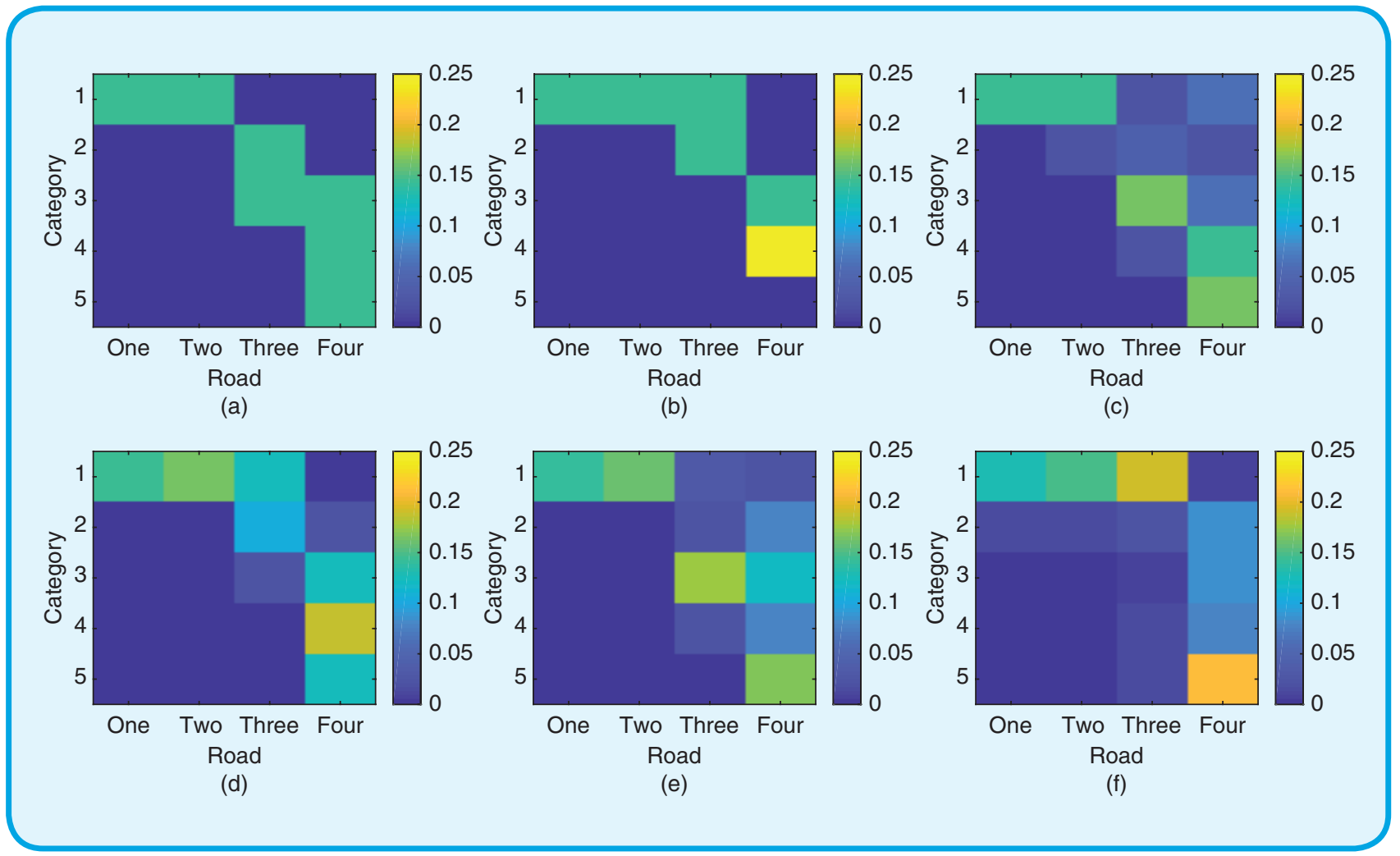

FIG 8 A comparison of the category result for the management function of planning (500 m) (a) DVI and (b) point laser system; programming (60 m), (c) DVI and (d) point laser system; and preparation $(20 \mathrm{~m}),(\mathrm{e}) \mathrm{DVI}$ and (f) point laser system. 


\section{Limited Road Information}

Road cracking is a common type of defect that occurs on local roads with asphalt surfacings. Therefore, for a comprehensive condition survey cracking needs to be detected since unchecked cracking can lead to the formation of potholes and eventual road failure. Road surface cracking can span both the transverse and longitudinal directions [31]. As shown in Figure 11, cracking in the transverse direction can be relatively easily detected by the advocated PL system. However, cracking in the longitudinal direction is less likely to be detected by the narrow laser beam.

Another significant defect that occurs on local roads is edge deterioration, which results from a lack of support and poorly draining shoulder material. This issue can soon worsen if the road edge is not repaired in a timely fashion. Since edge deterioration is wider than road cracking, it should be easily captured by a PL sensor, provided that the sensor aligns with the edge of the road. However, for the system proposed herein the PL is capable of measuring only the surface of the road underneath the vehicle. A potential solution to the measurement of both longitudinal cracking and edge wear is to employ sensors at an angle to the road surface so that the coverage can extend outside the vehicle. Also, PL sensors could be mounted away from the vehicle, on a retractable arm, for example, allowing a greater area to be covered. These additional PL sensors, however, will increase the cost of system hardware, and more data points need to be processed.

\section{Low-Level Features for Classification}

In comparison to the features determined by an MLS system (3D point cloud data), the 2D PL system provides fewer (lowerlevel) features. Although the PL system can detect road defects based on the variation in their depth, a single PL cannot assess on its own the width or shape of a road defect. For example, in Figure 11, cracking in the transverse direction has been scanned only a few times, which makes it difficult to assess

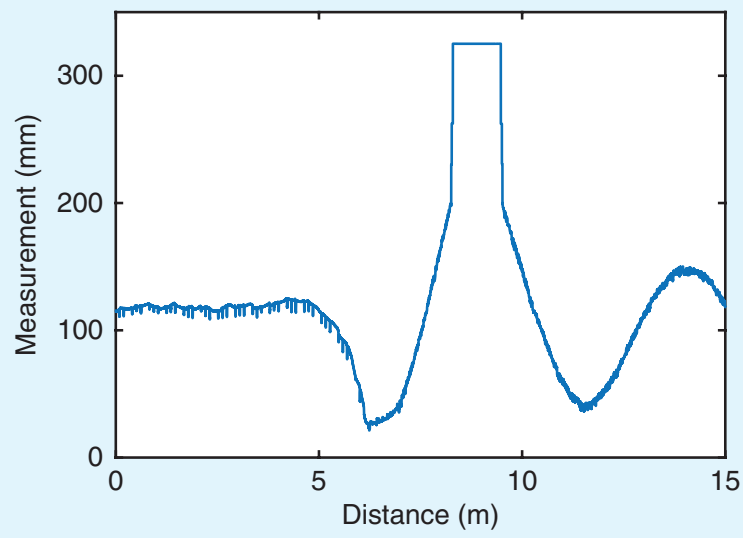

(a)

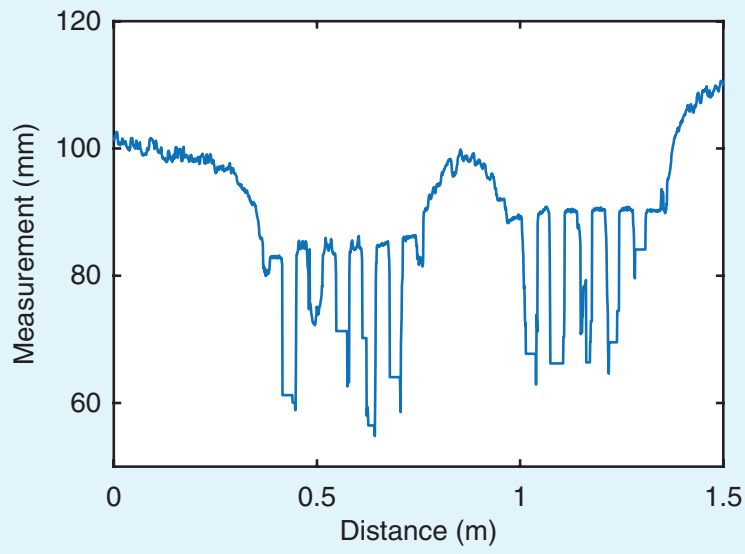

(c)

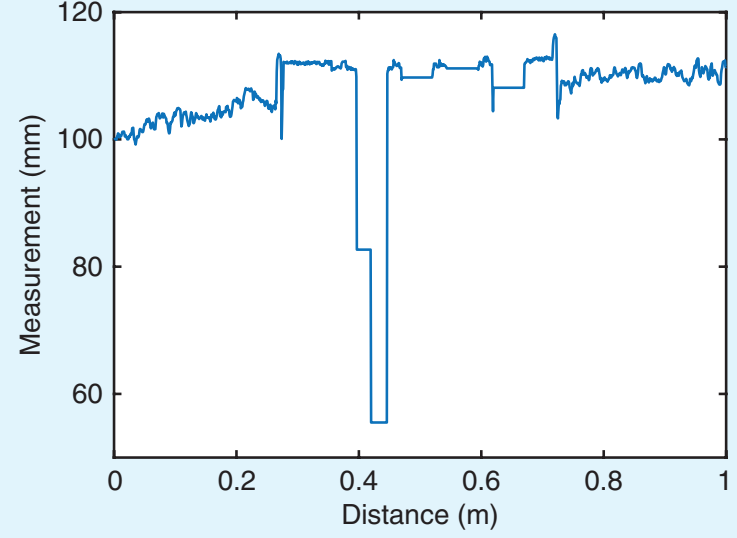

(b)

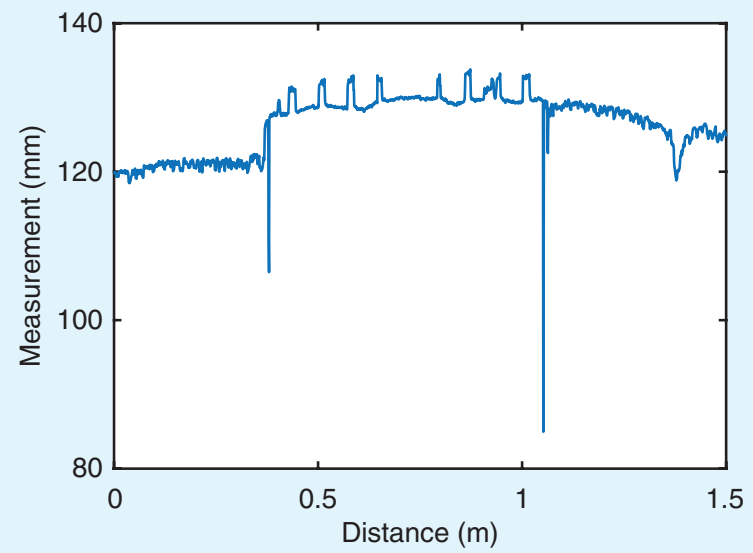

(d)

FIG 9 (a)-(d) Examples of different types of abnormal measurements from point laser sensor. 
the true extent of transverse cracking. This restricts the use of the PL system, reduces its ability to detect certain types of defects, and reduces the accuracy of defect classification compared to an MLS-based system. This could be improved by fusing data from more than one PL allied to the use of intelligent algorithms that can infer and extrapolate features from data provided.

In addition, the local-global principle requires finetuning if it is to be used to identify individual small defects, such as potholes. The longitudinal resolution of the PL sensor is $0.803 \mathrm{~mm}$ (see Table 1). Therefore, a pothole with a diameter of $30 \mathrm{~cm}$ would result in approximately 374 data points. This is a relatively small number when compared with a local length of $10 \mathrm{~m}(12,453$ data points $)$ and the

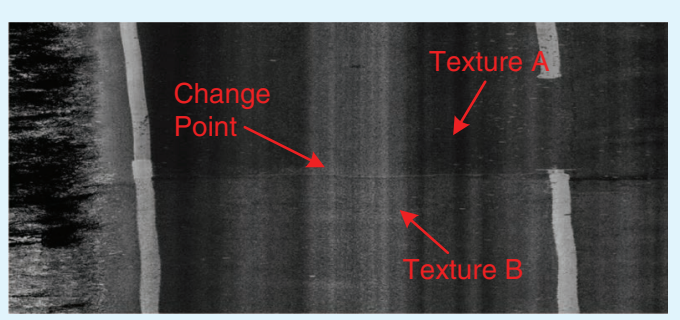

(a)

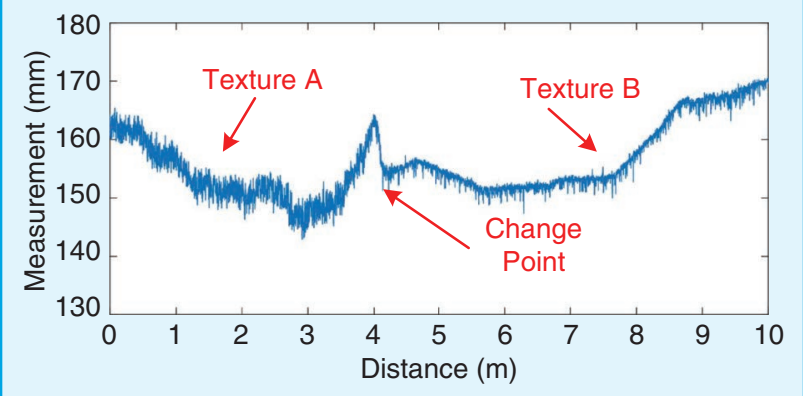

(b)

FIG 10 Data comparison between (a) a laser image from onboard MLS system and (b) the texture data from point laser system.

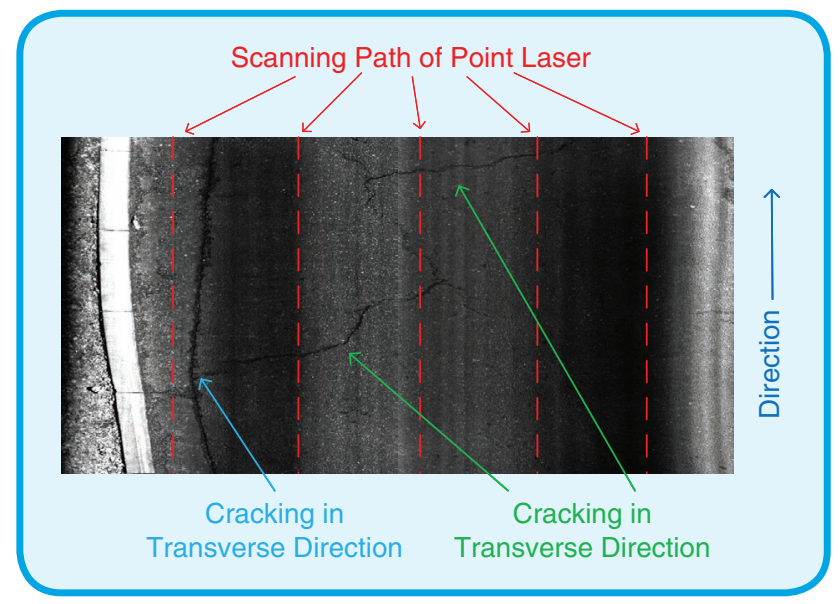

FIG11 A demonstration of a point laser scanning path. global length is $100 \mathrm{~m}(124,533$ data points). Accordingly, while the presence of the pothole would influence the histogram analysis described earlier and would probably result in local area of road surface being shown (correctly) to contain fretting, the analysis would not be able to identify the individual pothole causing the high degree of fretting. One possible solution is to use a moving window with variable size. For example, in our previous work [32], a peak detection approach was used, but it was of much greater computational complexity and required greater computational effort due to the wavelet transform process. Additionally, fusing data from multiple PL sensors will improve the feature diversity detectability of the suggested system by using the data from different scanning path.

\section{Conclusions}

This article discussed the use of a PL system for the automated analysis of local road condition at moving traffic speeds. A histogram analysis has been used to process the scanning data from a PL system with the local-global concept. The experimental results indicate that the PL system has the capability of satisfactorily assessing local road condition to IQL 3/4, which is required for strategic road asset management. However, several challenges were observed from the analysis of the field data, including the presence of unexpected measurements, issues associated with the change in road texture during a road survey, and the effects of vehicle dynamics. A number of corresponding solutions were proposed with respect to efficiency to identify noise and the use of the IMU to counteract the effects of vehicle dynamics on the recorded PL data. It is recognized that the proposed PL system is unable to detect a number of features that could be provided by a MLS system, thus limiting its application to the types of defects that can be detected and the IQL of those defects it is able to detect. It is recognized therefore that while the proposed PL system is able to assess road surface condition to a sufficient level to support strategic level road asset management, further enhancements are required to improve its detection performance in terms of accuracy and coverage and thereby enable road condition to be assessed to higher IQLs. These enhancements include fusing data from a number PLs allied to more intelligent data processing algorithms.

\section{Acknowledgments}

The authors are grateful for the financial support provided by the U.K. Technology Strategy Board through Innovate. The practical contribution of the Department of Civil Engineering at the University of Birmingham, U.K., is appreciated. We also gratefully acknowledge the support from Highways Surveyors Ltd and the Transport Research Laboratory (TRL) Ltd which provided road condition data captured using HARRIS2. 
About the Authors

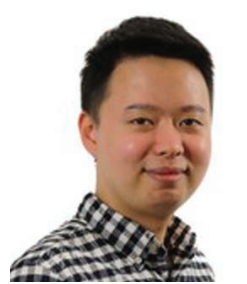

Wenda Li (w.li@bham.ac.uk) earned his Ph.D. degree in the Department of Electrical and Electronic Engineering at the University of Bristol. He is currently an honorary research fellow in the Department of Civil Engineering, University of Birmingham, Birmingham, B15 2TT, United Kingdom. His research interests include signal processing for radar-based applications, including passive Wi-Fi radar and penetrate ground radar. He is also interested in sensory signal analysis for autonomous vehicle and automatic road network assessment.

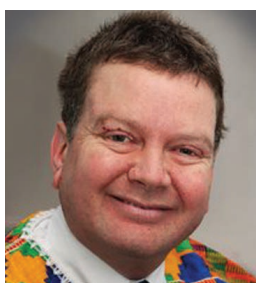

Michael Burrow (m.p.burrow@bham .ac.uk) earned his Ph.D. degree in road asset management from the University of Birmingham, Birmingham, B15 2TT, United Kingdom. He is a senior lecturer in the School of Engineering, University of Birmingham. His research interests include infrastructure asset management.

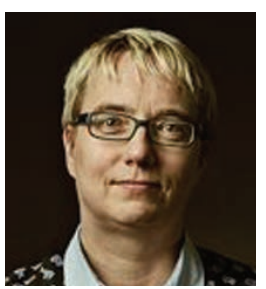

Nicole Metje (n.metje@bham.ac.uk) earned her Ph.D. degree from the University of Birmingham in 2001. She is a professor of infrastructure monitoring at the University of Birmingham, Birmingham, B15 2TT, United Kingdom. Her research focuses on detection of buried infrastructure and the development and use of sensing technologies to see through the ground and/or assess the condition of infrastructure assets.

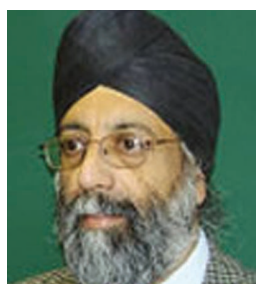

Gurmel Ghataora (g.s.ghataora@bham .ac.uk) earned his Ph.D. degree. in civil engineering from the University of Wales, Cardiff. He is currently a senior lecturer in the School of Civil Engineering, University of Birmingham, Birmingham, B15 2TT, United Kingdom. His research focuses on site investigation, materials testing (both laboratory and field), ground improvement, use of out-of-specification materials in construction, and improvement and design of both rural roads and railway track foundations.

\section{References}

[1] Road conditions in England: 2016. GOV.UK. Accessed: May 1, 2021. [Online]. Available: https://www.gov.uk/government/statistics/roadconditions-in-england-2016

[2] S. Mathavan, K. Kamal, and M. Rahman, "A review of three-dimensional imaging technologies for pavement distress detection and measurements," IEEE Trans. Intell. Transp. Syst., vol. 16, no. 5, pp. 2353-2362, 2015. doi: 10.1109/TITS.2015.2428655.
[3] M. E. Torbaghan, W. Li, N. Metje, M. Burrow, D. N. Chapman, and C. D. Rogers, "Automated detection of cracks in roads using ground penetrating radar," J. Appl. Geophys., vol. 179, p. 104,118, Aug. 2020. doi: 10.1016/j.jappgeo.2020.104118.

[4] S. C. Radopoulou and I. Brilakis, "Improving road asset condition monitoring," Transport. Res. Proc., vol. 14, pp. 3004-3012, 2016. [Online]. Available: https://www.sciencedirect.com/science/article/pii/ S2352146516304434, doi: 10.1016/j.trpro.2016.05.436.

[5] S. Sattar, S. Li, and M. Chapman, "Road surface monitoring using smartphone sensors: A review," Sensors, vol. 18, no. 11, p. 3845, 2018. doi: 10.3390/s18113845.

[6] C. Koch, K. Georgieva, V. Kasireddy, B. Akinci, and P. Fieguth, "A review on computer vision based defect detection and condition assessment of concrete and asphalt civil infrastructure," Adv. Eng. Inf., vol. 29, no. 2, pp. 196-210, 2015. doi: 10.1016/j.aei.2015.01.008.

[7] S. Paje, M. Bueno, F. Terán, U. Viñuela, and J. Luong, "Assessment of asphalt concrete acoustic performance in urban streets," J. Acoustical Soc. Amer., vol. 123, no. 3, pp. 1439-1445, 2008. doi: 10.1121/1.2828068.

[8] Y. Taniguchi, K. Nishii, and H. Hisamatsu, "Evaluation of a bicyclemounted ultrasonic distance sensor for monitoring road surface condition," in Proc. 7th Int. Conf. Comput. Intell., Commun. Syst. Netw. (CICSyN), 2015, pp. 31-34. doi: 10.1109/CICSyN.2015.16.

[9] D. Barber, J. Mills, and S. Smith-Voysey, "Geometric validation of a ground-based mobile laser scanning system," ISPRS J. Photogram. Remote Sens., vol. 63, no. 1, pp. 128-141, 2008. doi: 10.1016/j.isprsjprs.2007.07.005.

[10] H. Guan, J. Li, Y. Yu, M. Chapman, and C. Wang, "Automated road information extraction from mobile laser scanning data," IEEE Trans. Intell. Transp. Syst., vol. 16, no. 1, pp. 194-205, 2015. doi: 10.1109/ TITS.2014.2328589.

[11] M. Lehtomäki et al., "Object classification and recognition from mobile laser scanning point clouds in a road environment," IEEE Trans. Geosci. Remote Sens., vol. 54, no. 2, pp. 1226-1239, 2016. doi: 10.1109/ TGRS.2015.2476502.

[12] L. Zhang, F. Yang, Y. D. Zhang, and Y. J. Zhu, "Road crack detection using deep convolutional neural network," in Proc. 2016 IEEE Int. Conf. Image Process. (ICIP), pp. 3708-3712. doi: 10.1109/ICIP.2016.7533052.

[13] A. Zhang et al., "Automated pixel-level pavement crack detection on 3d asphalt surfaces using a deep-learning network," Comput.-Aided Civil Infrastruct. Eng., vol. 32, no. 10, pp. 805-819, 2017. doi: 10.1111/ mice.12297.

[14] H. Viner, P. Abbott, A. Dunford, N. Dhillon, L. Parsley, and C. Read, "Surface texture measurement on local roads," Transport Research Lab., Wokingham, Project Report PPR148, 2006.

[15] A. Wright, N. Dhillon, S. McRobbie, and C. Christie, "New methods for network level automated assessment of surface condition in the UK," in Proc. 7th Symp. Pavement Surface Characteristics, Norfolk, VA, Sept. $19-22,2012$.

[16] S. McRobbie, A. Wright, J. Iaquinta, P. Scott, C. Christie, and D. James, "Developing new methods for the automatic measurement of raveling at traffic-speed," in Proc. 11th Int. Conf. Asphalt Pavements, Nagoya, 2010.

[17] L. Ma, Y. Li, J. Li, C. Wang, R. Wang, and M. Chapman, "Mobile laser scanned point-clouds for road object detection and extraction: A review," Remote Sens., vol. 10, no. 10, pp. 1531, 2018. doi: 10.3390/rs10101531.

[18] "ProfiCura sensor family - 2D laser sensors for profile measurements." LIMAB. Accessed: May 1, 2021. [Online]. Available: http://www.limab .com/products/proficura-2d-2/

[19] "Laser crack measurement system." Pavemetrics. Accessed: May 1, 2021. [Online]. Available: http://www.pavemetrics.com/applications/ road-inspection/lcms2-en/

[20] Y. Yu, J. Li, H. Guan, F. Jia, and C. Wang, "Learning hierarchical features for automated extraction of road markings from 3-d mobile lidar point clouds," IEEE J. Sel. Topics Appl. Earth Observ. Remote Sens., vol. 8, no. 2, pp. 709-726, 2015. doi: 10.1109/JSTARS.2014.2347276.

[21] L. Lindner et al., "Mobile robot vision system using continuous laser scanning for industrial application," Ind. Robot, Int. J., vol. 43, no. 4, pp. 360-369, 2016. doi: 10.1108/IR-01-2016-0048.

[22] W. Li, M. Burrow, N. Metje, and G. Ghataora, "Automatic road survey by using vehicle mounted laser for road asset management," IEEE Access, vol. 8, pp. 94,643-94,653, May 2020. doi: 10.1109/ ACCESS.2020.2994470.

[23] S. E. Said and D. A. Dickey, "Testing for unit roots in autoregressivemoving average models of unknown order," Biometrika, vol. 71, no. 3, pp. 599-607, 1984. doi: 10.1093/biomet/71.3.599.

[24] W. Li, M. Burrow, and Z. Li, "Automatic road condition assessment by using point laser sensor," in Proc. 2018 IEEE Sensors, pp. 1-4. doi: 10.1109/ICSENS.2018.8589855.

[25] "Chapter 8: Detailed visual inspection (DVI)." UK Roads Liaison Group. Accessed: May 1, 2021. [Online]. Available: http://www 
.ukroadsliaisongroup.org/en/utilities/document-summary. cfm?docid=6C39F16D-B012-43BB-956247C5DFCD10F6

[26] R. Robinson, "Restructuring road institutions, finance and management: volume 1: concepts and principles,” Transportation Research Board, Washington, D. C., 2008. [Online]. Available: https://trid.trb.org/view/1152293

[27] "World bank data requirements." https://documents1.worldbank .org/curated/en/431821468340248050/pdf/373670trn0300data0 collection01PUBLIC1.pdf

[28] H. A. David, The Method of Paired Comparisons, vol. 12. London: C. Griffin, 1963.

[29] P. Malhotra, L. Vig, G. Shroff, and P. Agarwal, "Long short term memory networks for anomaly detection in time series," in Proc. 23rd Eur. Symp. Artif. Neural Netw., Comput. Intell. Mach. Learn., 2015, p. 89.
[30] Z.-M. Zhang, S. Chen, and Y.-Z. Liang, "Baseline correction using adaptive iteratively reweighted penalized least squares," Analyst, vol. 135, no. 5, pp. 1138-1146, 2010. doi: 10.1039/b922045c.

[31] J. Rolt, A. Otto, and A. A. Endale, "Common surface defects in asphalt road pavements and unexpected causes," in Proc. Inst. Civil Eng.Transport, 2018, pp. 1-8.

[32] W. Li, M. Burrow, N. Metje, Y. Tao, and G. Ghataora, "A novel processing methodology for traffic-speed road surveys using point lasers," IEEE Trans. Intell. Transp. Syst., vol. 22, no. 1, pp. 307-318, Jan. 2021. doi: 10.1109/TITS.2019.2957238. 